\title{
Leveraging Spatial Reuse with Adaptive Carrier Sensing in 802.11 Wireless Networks
}

\author{
Qiang Shen ${ }^{\ddagger}$, Xuming Fang ${ }^{\dagger}$ \\ ${ }^{\top}$ Provincial Key Lab of \\ Information Coding and Transmission \\ Southwest Jiaotong University \\ Chengdu, 610031 P. R. China \\ q.shen@ufl.edu,xmfang@swjtu.edu.cn
}

\author{
Rongsheng Huang $\ddagger$, Pan $\mathrm{Li}^{\ddagger}$ and \\ Yuguang Fang \\ ¥Department of ECE \\ University of Florida, Gainesville, FL 32611 USA \\ \{rshuang@, lipanleo@, \\ fang@ece.\}ufl.edu
}

\begin{abstract}
Recent studies indicate that by improving the spatial reuse ratio the throughput of 802.11 wireless networks can be improved. In this paper, we study the impact of physical carrier sensing and channel rate on the throughput of 802.11 wireless networks with chain topology. Firstly, this paper proposes that by adopting different carrier sensing thresholds for the transmissions of RTS and CTS, the blocking problem caused by exposed terminals is alleviated significantly. In 802.11 wireless networks with this modification, the spatial reuse ratio under certain channel rates can be increased to $\frac{1}{3}$, which is the highest value to our best knowledge. Secondly, in multirate networks, we demonstrate that $\frac{1}{3}$ is still the best value of special reuse ratio in terms of maximizing the achievable data rate. Thirdly, this paper proposes a new way to address the intraflow contention by decreasing the carrier sensing threshold of the source node. This method has shorter response time than that of the traditional method with adjusting the backoff window size. Finally, extensive simulations are implemented in NS2. The results show that our scheme significantly improves the throughput of 802.11 wireless networks with chain topology.
\end{abstract}

\section{Categories and Subject Descriptors}

C.2.1 [Network Architecture and Design]: Wireless Communications

\section{General Terms}

Algorithms, Design, Performance

\section{Keywords}

Medium Access Control (MAC), spatial reuse, carrier sensing, interference

\section{INTRODUCTION}

Wireless mesh networking is a promising scheme to access the Internet. It is widely deployed in many scenarios, such as campus

QShine 2008, July 28-31, 2008, Hong Kong, Hong Kong. Copyright 2008 ICST ISBN 978-963-9799-26-4

DOI 10.4108/ICST.QSHINE2008.3840 networking, community networking and so on. As a special form of wireless mesh networks (WMNs), wireless networks with chain topology have a simple architecture and a different application environment. For example, they are used in outdoor environment and their architectures usually appear to be a chain topology. Those features bring many advantages to the protocol design of this kind of networks. Protocols in wireless networks with chain topology can be designed specifically, so that they can be more efficient than the common protocols for universal topology.

Fig. 1 is one of the most common solutions to provide Internet access for the subscribers in vehicles [1]. It is a typical application of wireless networks with chain topology. Meanwhile, wireless networks with chain topology are also suitable for many other applications. Generally speaking, in those scenarios, gateways are several hops away from each other.

As the emergence of a variety of bandwidth demanding applications, the issue of how to increase the network throughput has received much attention. The network throughput depends on the achievable data rate at each individual wireless link. In fact, the achievable data rate relies on the sending rate, which is determined by the Signal-to-Interference-and-Noise-Ratio (SINR) at the receiver, and the spatial reuse relates to the total number of concurrent transmissions accommodated in the network. In 802.11 media access control (MAC) scheme, whenever a wireless node intends to transmit, it senses the channel and defers the transmission if the channel is busy. One can increase the level of spatial reuse either by reducing the transmit power or by increasing the carrier sensing threshold (thereby reducing the carrier sensing range) [2]. In this paper, we discuss how to increase the network throughput by adjusting spatial reuse in 802.11 wireless networks with chain topology.

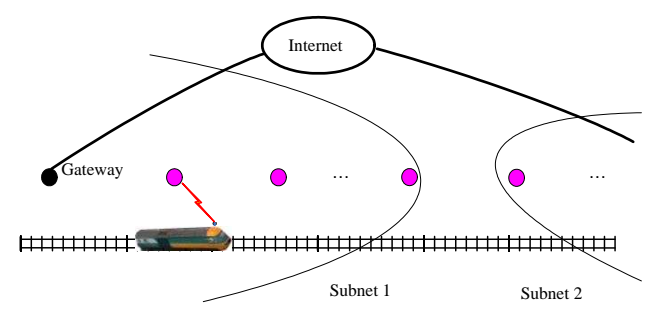

Figure 1: Typical application of wireless networks with chain topology

The rest of the paper is organized as follows. Section 2 presents the related work in wireless multi-hop networks. Section 3 introduces the wireless network models used in this paper. Then, Section 4 proposes a feasible scheme to address the blocking prob- 
lem caused by exposed terminals. Section 5 extends our proposed scheme to a multirate environment and derives the best concurrent transmission distance which can be used to maximize the achievable data rate. After that, we illustrate in Section 6 that by decreasing the sensing threshold, the intraflow contention can be alleviated. Finally, Section 7 provides the performance evaluation and Section 8 concludes our work.

\section{RELATED WORK}

Many papers have already discussed the impact of carrier sensing and spatial reuse on the system performance in multihop wireless networks. Zhai and Fang [3] investigate the impacts of SINR, topology, hidden/exposed terminal problems and bidirectional handshakes on the throughput of multirate and multihop wireless ad hoc networks. The authors conclude that a single value of physical carrier sensing range should be used for all the channel rates. Kim et. al. [2] derive that the achievable data rate follows the Shannon capacity and the spatial reuse depends on the ratio of the transmit power to the carrier sensing threshold. Based on this result, they propose a decentralized power and rate control algorithm to enable each node to adjust its transmit power and channel rate. Then, Lin et. al. [4] consider the issue of tuning PHY characteristics (transmit power and channel rate) and MAC parameters (backoff window size) jointly in unified framework in order to optimize the overall network throughput. However, all previous works use the Honey-grid model [5] which is widely used in ad hoc networks analysis.

Several works have focused on the chain topology. Guo et. al. [6] derive the minimum separation distance between simultaneous cochannel transmitters while maintaining a desirable SINR at the receivers. However, they only consider the directional transmissions and an ideal MAC protocol is required to maintain the optimal transmitter separation distance while minimizing the interference [7] In [8], Li et. al. claim that an ideal MAC protocol could achieve a spatial reuse ratio as much as $\frac{1}{3}$ in a chain topology theoretically. However, they do not provide any feasible solution to achieve this. The motivation of this paper is to design a feasible solution to achieve such a value by analyzing the SINR and the environment in 802.11 wireless networks with chain topology.

In addition, intraflow contention is another crucial problem in a chain topology. Zhai and Fang [9] introduce the intraflow contention in detail and conclude that by using different backoff window sizes for the source and other nodes, this problem can be addressed. Instead of adjusting the backoff window size, we address the intraflow contention problem by adjusting the carrier sensing threshold in this paper.

\section{WIRELESS NETWORK MODELS}

To analyze the maximum accumulated interference with various spatial reuse, wireless network models are discussed in this section. In addition, our analysis is based on the assumption that all the nodes use the same transmit power and only considers the traditional 802.11 MAC DCF with RTS/CTS.

\subsection{Physical layer model}

In real environments, the signal can be attenuated by several factors, including pathloss, multipath fading and shadowing [10]. Pathloss model [12] [13] is commonly used to describe the radio propagation property in wireless networks. In this paper, we only consider the signal attenuation caused by pathloss and use pathloss model as the physical layer model. Specifically, the received power, $P_{r x}\left(d_{t}\right)$, at any distance $d_{t}>d_{0}\left(d_{0}\right.$, usually 1 meter), can be ex-
Table 1: Signal-To-Noise Ratio and Receiver Sensitivity

\begin{tabular}{|c|c|c|}
\hline Rates $\left(r_{c}, \mathrm{Mbps}\right)$ & $S_{0}(\mathrm{~dB})$ & Receiver sensitivity $(\mathrm{dBm})$ \\
\hline 54 & 24.56 & -65 \\
\hline 48 & 24.05 & -66 \\
\hline 36 & 18.80 & -70 \\
\hline 24 & 17.04 & -74 \\
\hline 18 & 10.79 & -77 \\
\hline 12 & 9.03 & -79 \\
\hline 9 & 7.78 & -81 \\
\hline 6 & 6.02 & -82 \\
\hline
\end{tabular}

pressed in terms of the received power $\left(P_{0}\right)$ at $d_{0}$ :

$$
P_{r x}\left(d_{t}\right)=P_{0}\left(\frac{d_{0}}{d_{t}}\right)^{\gamma}
$$

The value of $P_{0}$ can be measured in the radio environment by taking the average received power at any point located at a close-in radial distance $d_{0}$ from a transmitter (Tx). $\gamma$ is the pathloss exponent that characterizes how quickly a signal fades in a particular network environment, and it usually ranges between 2 and 4 ( $\gamma=2$ for a free-space line-of-sight model and $\gamma=4$ for the tworay model). Specifically, since the wireless networks with chain topology (Fig. 1) are usually deployed in an outdoor or rural environment and the transmission model is the two-ray model, the typical value of $\gamma$ in [10] is 4 .

The energy detected by a receiver $(\mathrm{Rx})$ consists of three parts: intended signal from Tx, aggregate interference (from other concurrent transmitters, denoted by $\left.P_{I}\right)$ and background noise $\left(P_{N}\right)$. A successful reception must satisfy the following rules:

$$
\begin{gathered}
P_{r x}\left(d_{t}\right) \geq P_{R} \\
S I N R=\frac{P_{r x}\left(d_{t}\right)}{P_{I}+P_{N}}=\frac{P_{r x}\left(d_{t}\right)}{\sum_{i} P_{r x}\left(d_{i}\right)+P_{N}} \geq S_{0}
\end{gathered}
$$

where, $P_{R}$ is the receiver sensitivity. $S_{0}$ is the threshold of $S I N R$ for a correct perception, which is associated with the channel rate in 802.11 wireless networks. Normally, a higher channel rate requires a higher $S_{0}$ and the $S_{0}$ for different channel rate $\left(r_{c}\right)$ is provided in Table 1 [11].

\subsection{Interference model}

Considering 802.11 MAC, the minimal distance between the two transmission pairs is called separation distance illustrated in Fig. 2. Let $D$ denote the maximum transmission distance between two nodes. The definition of concurrent transmission distance $(k$ in hops) in this paper is one hop more than the separation distance. Hence, $\frac{1}{k}$ indicates the corresponding spatial reuse ratio. In this paper, we consider the chain topology illustrated in Fig. 3.

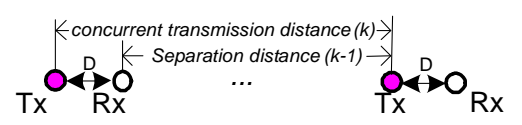

Figure 2: Definition of concurrent transmission distance in 802.11 wireless networks

In unidirectional transmission networks, in order to guarantee that all the concurrent transmissions can be conducted successfully, the aggregate interference at a receiver from all other concurrent transmissions should satisfy the restriction given by (3). In Fig. 3, 


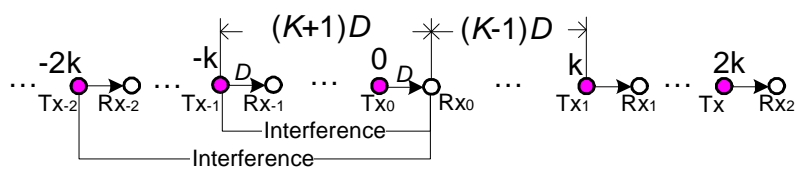

Figure 3: Interference model with unidirectional transmission

$P_{I}$ can be written as:

$$
\begin{aligned}
P_{I} & =\sum_{i=1}^{+\infty} P_{0}\left[\frac{d_{0}}{(i k-1) D}\right]^{\gamma}+\sum_{i=1}^{+\infty} P_{0}\left[\frac{d_{0}}{(i k+1) D}\right]^{\gamma} \\
& =P_{0}\left(\frac{d_{0}}{D}\right)^{\gamma} \sum_{i=1}^{+\infty}\left[\frac{1}{(i k-1)^{\gamma}}+\frac{1}{(i k+1)^{\gamma}}\right]
\end{aligned}
$$

If we consider the 802.11 MAC, the interference on the left side may come from $R_{X-1}, R_{X-2} \cdots$. Hence, (4) should be formulated as:

$$
P_{I}=P_{0}\left(\frac{d_{0}}{D}\right)^{\gamma} \sum_{i=1}^{+\infty}\left[\frac{1}{(i k-1)^{\gamma}}+\frac{1}{(i k)^{\gamma}}\right]
$$

Similar to other related analyses [2] [3], the closest interference node in each side contributes the majority of the interference. Hence, the interference to the tagged receiver can be approximated by:

$$
P_{I}=P_{0}\left(\frac{d_{0}}{D}\right)^{\gamma}\left[\frac{1}{(k-1)^{\gamma}}+\frac{1}{k^{\gamma}}\right]
$$

Since $P_{N}$ is much less than $P_{I}$ and $d_{t}=D$, (3) can be changed as follows:

$$
S I N R=\frac{P_{r x}(D)}{P_{I}}=\frac{1}{\frac{1}{k \gamma}+\frac{1}{(k-1) \gamma}} \geq S_{0}
$$

As described in Fig. 3, the interference from the left side is different from that of the right side. Since we take the 802.11 MAC into consideration, our interference model is more practical than the model described in [6].

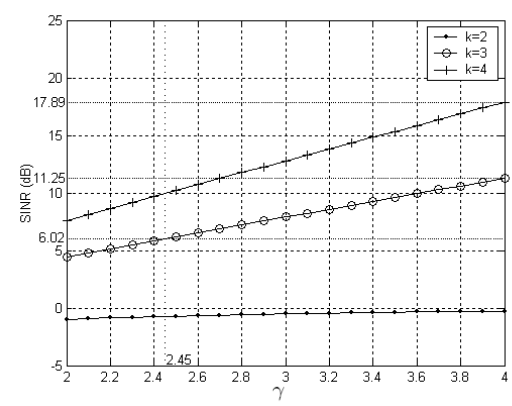

Figure 4: $S I N R$ with different $\gamma$ in terms of $k=\mathbf{2 , 3 , 4}$

$S I N R$ with different $\gamma$ in terms of $k=2,3,4$ are illustrated in Fig. 4 According to Fig. 4, we can observe that $k$ cannot be equal to 2 since the $S I N R$ is below the minimum $S_{0}(6.02 \mathrm{~dB})$ regardless the value of $\gamma$. Intuitively, when $k=2$, a receiver cannot distinguish the signal of one transmitter from that of another. When $k=3$, it is feasible that nodes which are 3 hops away from each other can transmit at the same time. Since $\operatorname{SINR}>6.02$ and $\gamma>2.45$, $k$ can be equal to 3 which collaborates the results of [3] and [6]. Furthermore, there are two important properties that deserve more attention (assume $k=3$ ):

\subsubsection{IEEE 802.11 MAC protocol limitation}

We use the topology shown in Fig. 5 to explain the problem. The distance between every two neighbors is $D$. Theoretically, node 1 and 4 can transmit at the same time when $k=3$. However, with the traditional 802.11 MAC, node 4's transmission can interfere with the RTS transmission from node 1 to node 2 . Although node 2 can capture the RTS frame from node 1, it cannot transmit CTS due to the busy channel. Namely, node 4's transmission blocks node 2's CTS transmission and we call it blocking problem hereafter. This problem will be addressed in Section 4.

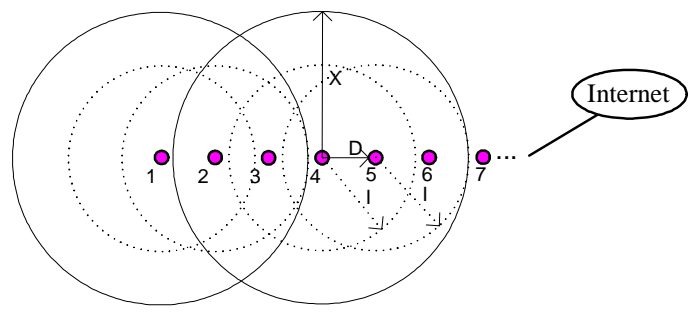

Figure 5: Typical chain topology

\subsubsection{Path loss exponent requirement}

$k=3$ is feasible only when $S I N R$ is bigger than $S_{0}$. Hence, when $S_{0}=6.02 \mathrm{~dB}$, there will be no doubt we can guarantee that $k=3$ if $\gamma>2.45$. However, if $\gamma=4$ and $k$ is equal to 3 and 4, respectively, the values of $S I N R$ are 11.25 and $17.89 \mathrm{~dB}$, respectively. According to Table 1, 18 and 24 Mbps can be supported with the constrain of the bit error rate. However, if we decrease the value of $k$, the number of concurrent transmissions can be increased. On the other hand, if we increase the value of $k$, the maximum channel rate that can be supported by the network will be increased due to the increase of SINR. Therefore, there should be a $k$ which can maximize the achievable data rate. We will study this problem in Section 5.

\section{SOLUTION TO THE BLOCKING PROB- LEM}

A node starts to transmit only when the level of sensing energy is below a carrier sensing threshold. A carrier sensing threshold determines how much interference a communication can tolerate and reflects a carrier sensing range $(X)$. Hereafter, we will discuss $X$ instead of the carrier sensing threshold. By analyzing the relationship between $X$ and the interference range $(I)$, we propose a feasible solution to alleviate the blocking problem. The following discussion in this section assume that the topology illustrated in Fig. 5 and $k=3$. We only focus on the minimum channel rate $(6 \mathrm{Mbps})$ in this section. Hence, $S_{0}$ in $\mathrm{dB}$ is equal to 6.02 and we will discuss other situations in the following sections.

\subsection{Optimum sensing range for RTS}

Within the interference distance any sender can ruin the reception of the tagged receiver. $I$ is given by:

$$
I=D\left[\frac{1}{S_{0}}-\left(\frac{D}{d_{0}}\right)^{\gamma} \frac{P_{N}}{P_{0}}\right]^{-\frac{1}{\gamma}}
$$

With negligible $P_{N},(8)$ becomes:

$$
I=D S_{0}{ }^{\frac{1}{\gamma}}
$$

Taking transmission from node 4 to node 5 for example, to keep the nodes in node 5's interference range silence, the sensing range 
of node 4 must cover the entire interference range of node 5 . Hence,

$$
X \geq D+I
$$

$X$ cannot be too large since it is related to the number of concurrent transmissions. Hence, we have the following limitation:

$$
X<k D
$$

When $k=3, I$ must be bigger than $D$ since a receiver cannot distinguish the signal of the tagged transmitter from that of the interference node. Finally, we can obtain the optimal carrier sensing range for the RTS transmission:

$$
2 D \leq X<3 D
$$

There will be no difference between $2 D$ and $3 D$, if the distance between two neighbors is equal to $D$. Finally, when $k$ appears to be anther value, the $X$ for RTS transmission is expressed as follows:

$$
(k-1) D \leq X<k D
$$

\subsection{Optimum sensing range for CTS}

Node 5 in Fig. 5 can receive RTS from node 4 successfully since $S I N R>S_{0}$ according to the analysis in section 3.2 when $\gamma>2.45$.

After receiving RTS, if the sensing range of node 5 is given the same power as used by node 4 . This phenomena is also observed in [8], node 5 fails to transmit CTS when node 7 is transmitting. However, according to our analysis in section 3.2, node 5's transmission will not interfere with other ongoing transmissions if $\gamma>2.45$ and $S_{0}=6.08 \mathrm{~dB}$. Furthermore, after the transmission of RTS, nodes in node 4's interference range will not initiate a new transmission in the duration of DIFS. Hence, while node 5 is transmitting CTS, node 3 and 6 will not transmit. Consequently, node 4 receives CTS successfully. Namely, node 4 receives CTS successfully since its $S I N R$ at this time is still higher than $S_{0}$ even when node 2 and 7 are transmitting at the same time.

Obviously, when node 5 is sending CTS, the CTS sensing range does not need to be the same as the RTS sensing range. In chain topology, since (7) is satisfied, the optimal sensing range for the receiver, when it sends CTS, is given by:

$$
D \leq X=I<2 D
$$

For the same reason as the RTS sensing range, there will be no difference if $X$ chooses different value between $D$ and $2 D$. Furthermore, when $k$ appears to be another value, the optimal $X$ for CTS transmission should be:

$$
(k-2) D \leq X<(k-1) D
$$

By adopting different carrier sensing ranges for the transmissions of RTS and CTS, node 4's transmission will not block node 2's CTS transmission anymore. Hence, the blocking problem can be alleviated. Meanwhile, $k$ can be guaranteed to be 3 and the spatial reuse ratio can reach the highest value in 802.11 wireless networks, namely, $\frac{1}{3}$, which is the highest spatial reuse to our best knowledge.

\section{MAXIMIZATION OF THE ACHIEVABLE DATA RATE}

In a multirate environment, a smaller $S I N R$ leads to a smaller channel rate. In this section, we try to study the relationship between the spatial reuse and the maximal achievable data rate $\left(r_{d}\right)$ which is the rate of the payload that we attempt to transmit.
Table 2: System parameters in IEEE 802.11

\begin{tabular}{|c|c|}
\hline Base Rate $\left(r_{b}\right)$ & $1 \mathrm{Mbps}$ \\
\hline SIFS $\left(T_{\text {sifs }}\right)$ & $10 \mu \mathrm{s}$ \\
\hline DIFS $\left(T_{\text {difs }}\right)$ & $50 \mu \mathrm{s}$ \\
\hline Backoff Slot time & $20 \mu \mathrm{s}$ \\
\hline Phy header $\left(L_{p h y}\right)$ & 192 bits \\
\hline MAC header $\left(L_{m a c}\right)$ & 256 bits \\
\hline Route header $\left(L_{r t}\right)$ & 160 bits \\
\hline Payload $\left(L_{p l}\right)$ & 8000 bits \\
\hline Data packet & $L_{p l}+L_{r t}+L_{m a c}+L_{p h y}$ \\
\hline RTS $\left(L_{r t s}\right)$ & 160 bits $+L_{p h y}$ \\
\hline CTS/ACK $\left(L_{c t s / a c k}\right)$ & 112 bits $+L_{p h y}$ \\
\hline
\end{tabular}

\subsection{Definition of the achievable data rate}

Given channel rate $r_{c}$, the corresponding $r_{d}$ can be obtained as described in [3]:

$$
r_{d}=\frac{1}{k} \frac{L_{p l}}{T_{b}+T_{p}+\frac{L_{H}+L_{p l}}{r_{c}}+T_{c}}
$$

where $T_{p}$ in seconds is a preamble of a packet regardless of the channel rate, such as the physical layer preamble for synchronization purpose at the receiver and the short interframe spacing $(S I F S)$ at the MAC layer. $T_{b}$ and $T_{c}$ are the average backoff time and the average collision time, accordingly. $L_{H}$ consists of protocol overheads in bits from different protocol layers, such as IP layer. $L_{p l}$ is the size of the payload in bits. If we adopt the minimum backoff window size (namely, 8 slots) and do not change it from time to time, $T_{b}$ will be equal to 4 slots. Due to addressing the blocking problem, $T_{c}$ is very small. Hence, we do not consider $T_{c}$ in this paper. Specifically, if the system parameters are given as described in Table $2, r_{d}$ can also be shown as:

$$
r_{d}=\frac{1}{k} \frac{L_{p l}}{T_{b}+\frac{L_{r t s}+L_{c t s}+L_{a c k}+L_{p h y}}{r_{b}}+3 T_{s i f s}+T_{d i f s}+\frac{L_{r t}+L_{m a c}+L_{p l}}{r_{c}}}
$$

$r_{d}$ is an estimated value of the accurate achievable data rate. According to our simulation results, we can verify that $r_{d}$ is an isotone mapping of the accurate achievable data rate.

\subsection{Relationship between the spatial reuse and the achievable data rate}

We use (7) to calculate the interference between nodes regarding different values of $k$. Once a node measures the accumulated interference, Table 1 can be used to calculate $r_{c}$ according to the level of SINR. Based on (16), $r_{d}$ can be obtained with the knowledge of $r_{c}$. Hence, we can get the relationship between $r_{d}$ and $r_{c}$ in terms of different $k$ as illustrated in Fig. 6.

Based on Fig. 6, we can conclude that when $\gamma=4$ and $r_{c}$ is 6 , 9,12 or $18 \mathrm{Mbps}, k=3$ is the best choice since a system with $k=3$ can provide the highest $r_{d}$ in a chain topology. However, a system with $k=3$ cannot support any higher $r_{c}$ because the requirement of $S I N R$ is so high and when $S I N R$ is below this value the bit error rate is not acceptable. If we want to use $r_{c}$ which is equal to 24,36 , 48 and 54 Mbps as the channel rate, the best choice of $k$ is 4, 5, 6 and 6 , accordingly.

To achieve the highest $r_{d}$ (1.5 Mbps), $k=3$ and $r_{c}=18 \mathrm{Mbps}$ is the best setting when $\gamma=4$. According to our numerical analysis, when $\gamma \geq 2.95$, a system with $k=3$ can still obtain higher $r_{d}$ than those with other values of $k$. Hence, in this paper, we demonstrate that $k=3$ is the best concurrent transmission distance to maximize $r_{d}$ when $\gamma>2.95$. In wireless networks with chain topology, 

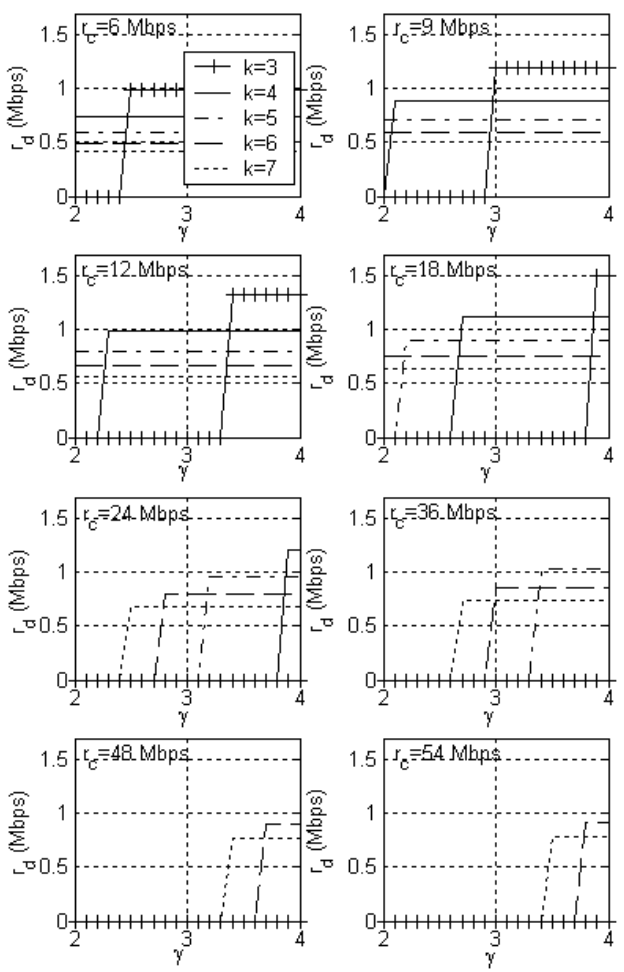

Figure 6: comparison of $r_{d}$ in the chain topology in terms of different $r_{c}$ and $k$

$\gamma>2.95$ can be satisfied easily since they are deployed in an outdoor or rural environment.

\section{ALLEVIATION OF THE INTRAFLOW CON- TENTION}

The nature of intraflow contention is that the source node and other nodes have different transmission probabilities. An intuition to address the intraflow contention is to adjust the transmission probability of each node to maximize the end-to-end throughput. In this section, we will investigate two ways of decreasing transmission probability of the source node: one is to differentiate various nodes by adopting different backoff window sizes and the other is to increase the carrier sensing range of the source node.

\subsection{Adjusting the backoff window size}

Adjusting the backoff window size is a traditional way to address the contention in wireless ad hoc networks. According to [9] [14], the size of the backoff window is very crucial to achieve the maximum throughput. The basic idea of this approach is to adjust the backoff window size to guarantee that all the nodes have the same probability to use channel resource.

When a node detects congestion, it notifies the upstream node to increase its backoff window size. When an upstream node receives the message, it uses different steps to increase and decrease the backoff window size, for example, increasing step is twice as long as the decreasing step. Hence, if congestion occurs, it can be alleviated immediately with the bigger step. When the wireless resource becomes abundant, the backoff window size is decreased with a smaller step. In this way, this scheme shortens the transmission delay and improves the efficiency of the resource usage.

\subsection{Increasing the sensing range}

As described in Fig. 5, if all the nodes in the network use the same carrier sensing range when $k=3,5$ nodes are in node 4's RTS sensing range and only 3 nodes in node 1's RTS sensing range. Since the number of nodes in the sensing range affects the transmission probability, node 1's transmission probability is much higher than that of node 4 . Therefore, we try to change the transmission probability of the source node by increasing its carrier sensing range in this subsection.

When there is a flow from node 1 to the gateway, we must prevent node 1 injecting more data packets to the network than that can be delivered immediately by the downstream nodes. To achieve this goal, the transmission probability of node 1 should be the same as those of node 3 and 4 . We try to find out the most suitable RTS sensing range for node 1 to alleviate the intraflow contention by experiment. The CTS sensing range here is still one hop less than the RTS sensing range. According to Fig. 7, we observe that when the RTS sensing range is bigger than 3 but smaller than 4 hops (denoted by 3.5 in Fig. 7), the best performance can be achieved.

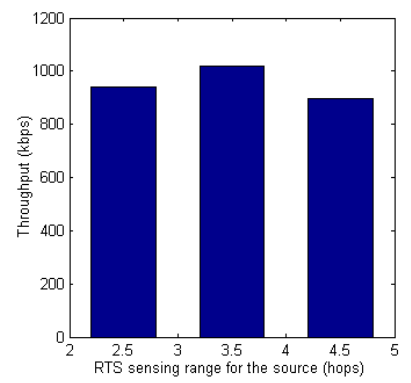

Figure 7: The maximum throughput with different RTS sensing ranges

We should notice that, using a larger sensing range by the source node does not mean that it can be allowed to transmit with a higher level of $r_{c}$. Instead, it still uses $r_{c}$ as the channel rate which is determined by the normal RTS sensing range as discussed in Section 5 . The shortcoming of this idea is that we can only adjust the sensing range to cover one more node or less. Hence, we cannot adjust it more precisely with a smaller step. Nevertheless, this method addresses the intraflow contention perfectly in a chain topology when $k=3$. In addition, if we consider multiflows in our scenario, nodes should use different sensing ranges for packets which are forwarded to guarantee that each flow has a fair probability to share the resource. However, this is beyond the scope of this paper, and we will discuss the fairness problem in our future work.

Adjusting the backoff window size to achieve the optimal throughput takes long time since the optimal backoff window size is determined by the traffic tensity [14]. In contract, increasing the sensing range takes no time since it is predetermined. Consequently, increasing the sensing range is better than adjusting the backoff window size in terms of the response time. The further comparison of the two methods will be discussed in the following section.

\section{PERFORMANCE EVALUATION}

All the simulations in this section are implemented in NS2. We do not use any special routing protocol. When a node receives a packet, it forwards the packet to the next predetermined hop.

\subsection{Alleviating the blocking problem}

We use the simple four nodes topology where $r=3$ and $r_{c}=6 \mathrm{Mbps}$ 


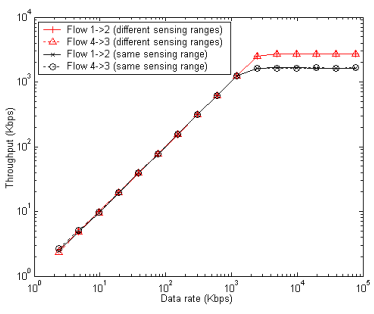

(a) Node 1 to 2 and 4 to 3

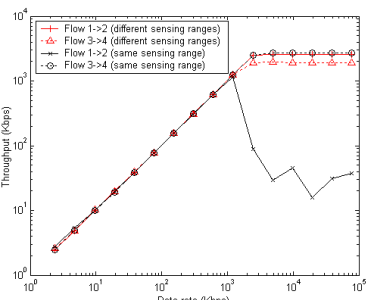

(b) Node 1 to 2 and 3 to 4
Figure 8: Solution to the blocking problem

to describe this problem. Node 1 and 2 are in each other's transmission range, so are node 3 and 4 . However, node 3 is two hops away from node 2. Namely, node 3 and 2 are in each other's RTS sensing range but not CTS range.

One flow is from node 1 to node 2 and another is from node 4 to node 3. The performance in Fig. 8(a) shows that two flows do not interfere with each other because of different RTS/CTS sensing ranges.

However, by changing the direction of one flow, there will be a little difference from the previous scenario in performance. If two flows can transmit simultaneously, two flows will not interfere with each other. In this paper, we do not assume the two flows are synchronized. Hence, node 3 cannot transmit RTS while node 2 is transmitting CTS since node 2 is in node 3's RTS sensing range. Consequently, flow 2 will be interfered by flow 1 . In contract, node 2 can transmit CTS while node 3 is transmitting RTS or DATA. Therefore, flow 1 will not be interfered by flow 2 . According to the performance shown in Fig. 8(b), our scheme can alleviate the blocking problem to a great deal.

\subsection{Achieving the maximum channel rate}

We demonstrate the maximum $r_{d}$ with different values of $k$, for example, 3, 4, 5 and 6. Fig. 6 is the theoretical analytical result. In this subsection, we try to verify it by NS2 simulations. According to our previous analysis, we set the basic channel rates as $18,24,36$ and $54 \mathrm{Mbps}$ for $k=3,4,5$ and 6, respectively. The sensing ranges for RTS and CTS are set as (13) and (15), accordingly.

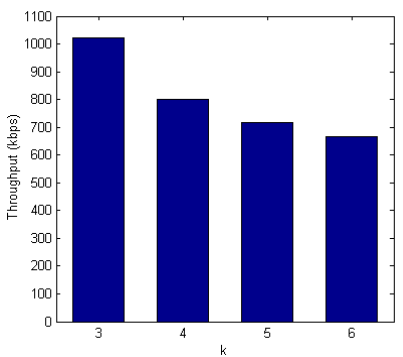

Figure 9: End-to-end throughput with different values of $k$

Fig. 9 illustrates the maximal achievable data rates when $k$ is chosen with different values. We can conclude that when $k=3$ the highest achievable data rate can be achieved, which is the same as the result obtained in Section 5. The maximal achievable data rate obtained in NS2 simulation is smaller than that of our previous theoretical result since we did not consider the collisions in our theoretical analysis.

\subsection{Alleviating the intraflow contention}

The simulations of this subsection are done in a chain topology with 7 nodes. When we alleviate intraflow contention by increasing the RTS sensing range of the source node (IRSR), the minimal backoff window size is used by all the nodes in the chain.

The fixed window size method which is the same as the one described in [9] is used to compare with our scheme. 32 is set to be the backoff window size by the source node and 8 by others, and all of them are fixed. To compare with our scheme, we implement a simple method to adjust the backoff window size dynamically based on the fixed window size method, namely, dynamically adjusting window size (DAW). Each node checks the length of its queue periodically, if it is larger than half of the total length, DAW increases the backoff window size of the upstream nodes. In contract, if it is smaller than a threshold (we use 4 slots in our simulation) and the backoff window size is larger than 8 slots, backoff window size of upstream node will be decreased. In addition, we set the length of transmitting queue, increasing step and decreasing step as 50,8 and 4 slots, respectively. Based on those methods, we can get those results shown in Fig. 10.

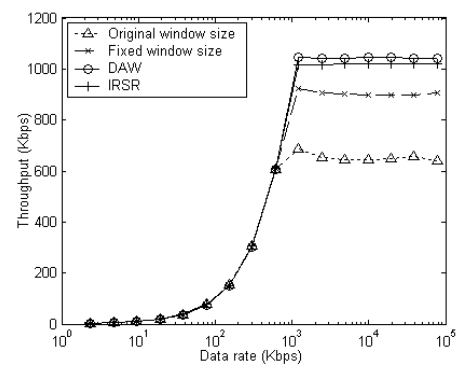

Figure 10: Average End-to-end throughput comparison of different methods

From Fig. 10, we can observe that both DAW and IRSR can alleviate the intraflow contention problem significantly. Due to the benefit of addressing the intraflow contention, the average end-toend throughput is absolutely higher than that without addressing this problem. Meanwhile, both of them are more efficient than the fixed window size method and the original backoff method in 802.11 MAC. Finally, since DAW is more precious than that of IRSR, its average throughput is a little better than that of IRSR.

The performance of each hop is shown in Fig. 11(a) when a fixed channel rate is used from the source to the destination. The performance of IRSR shows that there is no contention since the throughput of every hop is stable. Hence, IRSR prevents the intraflow contention from happening. For DAW, the throughput of first several hops is bigger than that of others. Namely, the packet loss ratio of first several hops must be higher than others. Consequently, DAW uses the resource efficiently, but its throughput is not as stable as that of IRSR. In addition, at the beginning, the throughput of IRSR is a little better than that of DAW.

The delays of DAW and IRSR are compared in Fig. 11(b). At the beginning, with IRSR, the intraflow contention does not affect the delays at all. With DAW, the delay is much bigger than that of IRSR at the beginning. In addition, the average delay of DAW is 1.15 times as much as that of IRSR.

Both of the two methods address the intraflow contention properly. However, IRSR is better in terms of the delay and throughput at the beginning. Hence, IRSR has shorter response time than that of DAW in 802.11 wireless networks with chain topology.

\section{CONCLUSIONS}




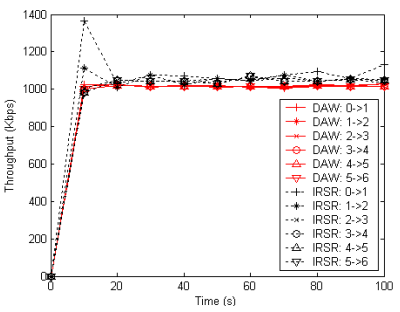

(a) Throughput

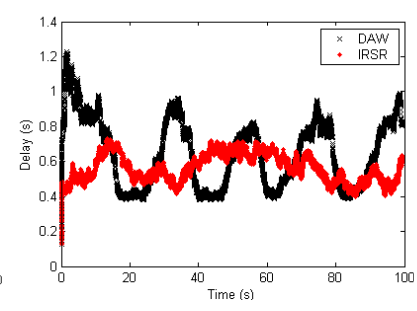

(b) Delay
Figure 11: Performance comparisons of IRSR and DAW

Increasing the throughput of 802.11 wireless networks is always a big challenge. In this paper, we study the impact of physical carrier sensing and channel rate on the throughput of the 802.11 wireless networks with chain topology. Extensive simulations show that, under certain channel rates, adopting different carrier sensing thresholds for the transmissions of RTS and CTS can achieve the highest spatial reuse ratio, i.e., $\frac{1}{3}$, which is the best spatial reuse ratio to maximize the achievable data rate when $\gamma>2.95$. Furthermore, by increasing the sensing range of the source node, our scheme alleviates greatly the intraflow contention problem and improves the throughput performance significantly.

\section{ACKNOWLEDGMENT}

The work of Qiang Shen and Xuming Fang was partially supported by the PhD innovation Fund of SWJTU, NSFC under Grant 60772085 and the Open Research Fund of State Key Lab of ISN, Xidian University under Grant ISN8-01. The work of Yuguang Fang was partially supported by Chunghwa Telecom M-Taiwan Program M-Taoyuan Project and the National Science Council (NSC), R.O.C., under the NSC Visiting Professorship with contract number NSC-96-2811-E-002-010 while he was a visiting professor in the Department of Computer Science and Information Engineering at National Taiwan University.

\section{REFERENCES}

[1] M. Xie, X. Fang, Q. Shen. "A Cross-layer Fast Handoff Protocol for Vehicle Communication System," In IET CCWMSN, Shang Hai, China, Oct. 2007.

[2] T. Kim, H. Lim and J. Hou, "Improving Spatial Reuse through Tuning Transmit Power, Carrier Sense Threshold, and Data Rate in Multihop Wireless Networks," In ACM MOBICOM, Los Angeles, USA, Sep. 2006.

[3] H. Zhai and Y. Fang, "Physical Carrier Sensing and Spatial Reuse in Multirate and Multihop Wireless Ad Hoc Networks," In IEEE Infocom, Barcelona, Spain, April 2006.

[4] T. Lin and J. Hou, "Interplay of Spatial Reuse and SINR-determined Data Rates in CSMA/CA-based, Multi-hop, Multi-rate Wireless Networks," In IEEE INFOCOM, 803-811, Anchorage, USA, May 2007.

[5] R. Hekmat and P. V. Mieghem, "Interference in Wirelwss Multi-hop Ad-hoc Networks and its Effect on Network capacity," Wireless Networks, 10(4):389-399, July 2004.

[6] X. Guo, S. Roy and W. S. Conner, "Spatial Reuse in Wireless Ad-hoc Networks," In IEEE VTC, 3:1437-1442, Orlando, USA, Oct. 2003.

[7] J. Zhu, X. Guo, L. L. Yang and W. S. Conner, "Leveraging Spatial Reuse in 802.11 Mesh Networks with Enhanced Physical Carrier Sensing," In IEEE ICC, 7:4004-4011. Paris, France, June 2004.
[8] J. Li, C. Blake, D. S. J. De Couto, H. I. Lee and R. Morris, "Capacity of Ad Hoc Wireless Networks," In ACM MOBICOM, Rome, Italy, July 2001.

[9] H. Zhai and Y. Fang, "Distributed Flow Control and Medium Access in Multihop Ad Hoc Networks," IEEE Tran. on Mobile Comp., 5(11):1503-1514, Nov. 2006.

[10] T. S. Rappaport, "wireless Communications: Principles and Practice (Second Edition)," Upper Saddles River Prentice-Hall, 2002.

[11] J. Yee and H. Pezeshki-Esfahani, "Understanding wireless lan performance trade-offs," CommsDesign.Com, 2002.

[12] P. Gupata and P. R. Kumar, "The Capacity of Wireless Networks," IEEE Tran. on Information Theory, 46(2):388-404, March 2000.

[13] T. J. Shepard, "A Channel Access Scheme for Large Dense Packet Radio Networks," In ACM SIGCOMM, 219-230, California, USA, Aug. 1996.

[14] F. Cali, M. Conti and E. Gregori, "Dynamic Tuning of the IEEE 802.11 Protocol to Achieve a Theoretical Throughtput Limit," IEEE Tran. on Networking, 8(6):785-799, Dec. 2000. 